\title{
Transition Disks - Grain Growth, Planets, or Photoevaporation?
}

\author{
T. Birnstiel ${ }^{1,2, a}$, P. Pinilla ${ }^{3}$, S. M. Andrews ${ }^{4}$, M. Benisty ${ }^{5}$, and B. Ercolano ${ }^{1,2}$ \\ ${ }^{1}$ University Observatory Munich, Scheinerstr. 1, D-81679 München, Germany \\ ${ }^{2}$ Excellence Cluster Universe, Technische Universität München, Boltzmannstr. 2, 85748 Garching, Germany \\ ${ }^{3}$ Institut für Theoretische Astrophysik, Universität Heidelberg, Albert-Ueberle-Str. 2, 69120 Heidelberg, \\ Germany \\ ${ }^{4}$ Harvard-Smithsonian Center for Astrophysics, 60 Garden Street, Cambridge, MA 02138, USA \\ ${ }^{5}$ Institut de Planétologie et d'Astrophysique de Grenoble (IPAG) UMR 5274, Grenoble, F-38041, France
}

\begin{abstract}
In the past years, many transition disks have been detected via SED modeling or imaging. The disks feature dust cavities in their inner regions with sizes ranging from a few to more than $70 \mathrm{AU}$. The origin of those structures, however, remains mysterious. We will present how our recent simulation results, which include gap opening by a giant planet and effects of dust evolution, could explain these structures.
\end{abstract}

\section{Introduction}

While today's instruments enable detailed studies of extrasolar planets, their statistics [1-3], or their atmospheres [4-6], their formation occurs hidden in the dense environments of protoplanetary disks. A particular class of protoplanetary disks, the so called transition disks, has been identified through a dip at mid-infrared wavelengths which could be modeled through a dearth of dust in the inner regions [7]. In recent years, (sub-)millimeter imaging has confirmed that these disks have a substantial deficit in their dust surface density in the inner regions [8-17].

These observations have been interpreted as being a signature of inside-out dispersal by photoevaporation [18-22], by opacity reductions caused by growing dust grains [23-26], or by structures caused by giant planets [27-29] or vortices [30,31]. Understanding the origin of these observations would be a milestone towards understanding the structure and evolution of protoplanetary disks and the formation of planets. In the following we will summarize some recent developments in this area of research and present new results which could explain the origin of transition disks.

\section{Prospects of the various mechanisms}

\subsection{Photoevaporation}

Observing a MIR dip in the SED is sufficient, but not necessary to reveal the transition feature of a disk. Imaging in the mm wavelength range is needed to conclusively determine whether or not a disk contains an inner cavity because relatively minor amounts of small dust are enough to produce

\footnotetext{
ae-mail: til.birnstiel@1mu.de
} 
a normal SED signature while still having a lack of dust surface density in the inner disk [17]. The current census of imaged protoplanetary disks is far from complete, but the sample of nearby, mmbright disks which have been observed at mm-wavelength show that the fraction of transition disks should be somewhere between 25 and $70 \%$ [17]. This fraction indicates that the life time of the transition phase of the disk is comparable to the disk life time. This is in contrast to the two-timescale behavior of photoevaporation models in which a disk vanishes quickly once the accretion rate through the disk has dropped to values which are comparable to the photoevaporative mass loss rates [18]. Transition disks seem to have somewhat lower accretion rates than standard disks [17, 32], but these rates are still too high to be explained by current X-ray and FUV photoevaporation models.

Lastly, the median hole size of those mm-bright disks is 35 AU with many cases beyond $70 \mathrm{AU}$ [17]. In contrast to this, the inner radius of holes created by photoevaporation is close to the gravitational radius, so typically around 2-3 AU. The subsequent inside-out dispersal of the disk is too fast to explain the large number of cases with large cavity radii [33].

Taken together, these facts make it seem very unlikely that photoevaporation can be the cause of all disks with inner cavities. However, it is possible that there are two populations of transition disks, one with low accretion rates and small inner holes and a second population of disks with large accretion rates and large cavity radii. This scenario is supported by the statistical analysis of [34].

\subsection{Grain Growth}

The time scales on which grain growth acts are proportional to the orbital time scale and the dustto-gas ratio [35-37]. It was therefore suggested that grain growth which occurs faster in the inner regions of the disk locks up most of the dust mass in large bodies which become effectively invisible to observations. Indeed, models of grain growth presented in [23] and [24] showed a dip in the mid-IR part of SEDs. However these models were only able to achieve this if grains would only stick and not fragment upon mutual collisions. Fragmentation acts as a source of small dust which effectively keeps the disks optically thick at visible wavelength [38].

In [36] and [39], we have shown that models of the dust size evolution and radial transport can lead to situations where dust fragmentation is kept at a low enough level and consequently, a dip in the IR is observed even though fragmentation is included in the model. However, these models are unable to produce large enough inner holes in the necessary time frame. Additionally, any opticaldepth declines seem to be too smooth to explain the observed sharp cavity edges. However, the shapes of the cavity edges are still under debate [40].

In summary, pure grain growth effects do not seem to be able to explain the observed signatures of transition disks.

\subsubsection{Planets \& Instabilities}

The possibility that transition disks are caused by a companion embedded in the disk is compelling and often quoted, however it has not been conclusively excluded or confirmed yet. Early models have been presented in [41] assuming a constant dust-to-gas ratio, but the physics of growth and transport of dust complicates this scenario. The challenge comes from the fact that transition disks can have very large, thoroughly-depleted cavities while still maintaining a significant accretion rate onto the central star. This cannot be explained even if multiple giant planets were to be embedded in the disk [29].

The possible solution to this is called "dust-filtration": in this picture, a single planet disturbs the gas disk causing not only a gap in the gas disk around the planet, but also a pressure maximum which 
can be significantly further outside the planets radius (e.g., 10 Hill radii for a planet five times the mass of Jupiter). Such a pressure maximum could also be produced by a large scale vortex [31, 42]. Grains which have grown to sizes of about a millimeter to a meter react sensitively to the pressure gradient of the disk, i.e. they move towards pressure maxima [43, 44]. This way, dust drifting from the outside-in gets trapped at the position of the pressure maximum. Gas on the other hand can flow past the planet, apart from the fraction which is accreted onto the planet. Therefore, the disk inward of the planet is still resupplied by gas, but cut-off from its resupply of dust. The mobility of dust allows a quick formation of the inner cavity and at the same time a long life time of the trapped dust outside of the planets orbit.

Shattering dust collision can redistribute some of the dust mass from the large grains into small dust which is well coupled to the gas flow. This opens a path for a small fraction of the dust to be transported into the inner regions. Which sizes can be transported this way depends on the shape of the surface density at the disk edge, the planets mass, and the details of the coagulation / fragmentation cycle.

\section{Summary and conclusions}

In this paper, we have summarized some of the recent attempts of modeling and explaining the origin of transition disks, i.e., disks with large inner dust cavities. We have discussed that both photoevaporation and grain growth are unable to explain all the observed signatures of transition disks. Large scale pressure maxima, which can be produced either by a giant planet embedded in the disk or by large scale vortices are able to filter large dust grains and allow only accretion of small dust. This way, the inner disk quickly becomes devoid of large dust - in agreement with mm imaging - while the outer disk can retain large grains by trapping them in the pressure maximum. The amount of small dust that is produced by fragmentation events and the details of the flow pattern around the planet determine how much fine-grained material is entering the inner disk.

Future work will focus on the details of this filtration effect, how it depends on the corotation region, and how its implications can be further tested by observations.

\section{References}

[1] S. Ida, D.N.C. Lin, ApJ 719, 810 (2010)

[2] C. Mordasini, Y. Alibert, W. Benz, H. Klahr, T. Henning, A\&A 541, 97 (2012)

[3] A.W. Howard, G.W. Marcy, S.T. Bryson, J.M. Jenkins, J.F. Rowe, N.M. Batalha, W.J. Borucki, D.G. Koch, E.W. Dunham, T.N. Gautier et al., ApJS 201, 15 (2012)

[4] J.L. Bean, E.M.R. Kempton, D. Homeier, Nature 468, 669 (2010)

[5] M. Brogi, I.A.G. Snellen, R.J. de Kok, S. Albrecht, J. Birkby, E.J.W. de Mooij, Nature 486, 502 (2012)

[6] A. Lecavelier Des Etangs, V. Bourrier, P.J. Wheatley, H. Dupuy, D. Ehrenreich, A. Vidal-Madjar, G. Hébrard, G.E. Ballester, J.M. Désert, R. Ferlet et al., A\&A 543, L4 (2012)

[7] C. Espaillat, N. Calvet, P. D’Alessio, E. Bergin, L. Hartmann, D. Watson, E. Furlan, J. Najita, W. Forrest, M. McClure et al., ApJ 664, L111 (2007)

[8] V. Piétu, A. Dutrey, S. Guilloteau, E. Chapillon, J. Pety, A\&A 460, L43 (2006)

[9] A.M. Hughes, D.J. Wilner, N. Calvet, P. D’Alessio, M.J. Claussen, M.R. Hogerheijde, ApJ 664, 536 (2007)

[10] J.M. Brown, G.A. Blake, C. Qi, C.P. Dullemond, D.J. Wilner, ApJ 675, L109 (2008) 
[11] A.M. Hughes, S.M. Andrews, C. Espaillat, D.J. Wilner, N. Calvet, P. D’Alessio, C. Qi, J.P. Williams, M.R. Hogerheijde, ApJ 698, 131 (2009)

[12] S.M. Andrews, D.J. Wilner, A.M. Hughes, C. Qi, C.P. Dullemond, ApJ 700, 1502 (2009)

[13] J.M. Brown, G.A. Blake, C. Qi, C.P. Dullemond, D.J. Wilner, J.P. Williams, ApJ 704, 496 (2009)

[14] A. Isella, J.M. Carpenter, A.I. Sargent, ApJ 714, 1746 (2010)

[15] S.M. Andrews, D.J. Wilner, A.M. Hughes, C. Qi, C.P. Dullemond, ApJ 723, 1241 (2010)

[16] A. Isella, A. Natta, D. Wilner, J.M. Carpenter, L. Testi, ApJ 725, 1735 (2010)

[17] S.M. Andrews, D.J. Wilner, C. Espaillat, A.M. Hughes, C.P. Dullemond, M.K. McClure, C. Qi, J.M. Brown, ApJ 732, 42 (2011)

[18] C. Clarke, A. Gendrin, M. Sotomayor, MNRAS 328, 485 (2001)

[19] R.D. Alexander, C. Clarke, J.E. Pringle, MNRAS 369, 216 (2006)

[20] U. Gorti, C.P. Dullemond, D. Hollenbach, ApJ 705, 1237 (2009)

[21] B. Ercolano, J.J. Drake, J.C. Raymond, C.C. Clarke, ApJ 688, 398 (2008)

[22] J.E. Owen, B. Ercolano, C.J. Clarke, R.D. Alexander, MNRAS 401, 1415 (2010)

[23] C.P. Dullemond, C. Dominik, A\&A 434, 971 (2005)

[24] H. Tanaka, Y. Himeno, S. Ida, ApJ 625, 414 (2005)

[25] J.R. Najita, N. Crockett, J.S. Carr, ApJ 687, 1168 (2008)

[26] K.M. Pontoppidan, G.A. Blake, E.F. van Dishoeck, A. Smette, M.J. Ireland, J. Brown, ApJ 684, $1323(2008)$

[27] W.K.M. Rice, K. Wood, P.J. Armitage, B.A. Whitney, J.E. Bjorkman, MNRAS 342, 79 (2003)

[28] S. Wolf, Physica Scripta 130, 4025 (2008)

[29] Z. Zhu, R.P. Nelson, L. Hartmann, C. Espaillat, N. Calvet, ApJ 729, 47 (2011)

[30] S. Wolf, H. Klahr, ApJ 578, L79 (2002)

[31] Z. Regály, A. Juhász, Z. Sándor, C.P. Dullemond, MNRAS 419, 1701 (2012)

[32] J.R. Najita, S.E. Strom, J. Muzerolle, MNRAS 378, 369 (2007)

[33] J.E. Owen, B. Ercolano, C.J. Clarke, MNRAS 412, 13 (2011)

[34] J.E. Owen, C.J. Clarke, MNRAS 426, L96 (2012)

[35] F. Brauer, C.P. Dullemond, T. Henning, A\&A 480, 859 (2008)

[36] T. Birnstiel, H. Klahr, B. Ercolano, A\&A 539, 148 (2012)

[37] S. Okuzumi, H. Tanaka, H. Kobayashi, K. Wada, ApJ 752, 106 (2012)

[38] T. Birnstiel, C.P. Dullemond, F. Brauer, A\&A 503, L5 (2009)

[39] T. Birnstiel, S.M. Andrews, B. Ercolano, A\&A 544, 79 (2012)

[40] A. Isella, L.M. Pérez, J.M. Carpenter, ApJ 747, 136 (2012)

[41] S. Wolf, G. D’Angelo, ApJ 619, 1114 (2005)

[42] W. Lyra, A. Johansen, H. Klahr, N. Piskunov, A\&A 493, 1125 (2009)

[43] F.L. Whipple, From Plasma to Planet p. 211 (1972)

[44] S.J. Weidenschilling, MNRAS 180, 57 (1977) 